\title{
PREFACE: NON-NEWTONIAN DROPS AND SPRAYS
}

The interest in non-Newtonian sprays has increased considerably in recent years, driven by their relevance to applications such as coating and painting, spray drying, additive manufacturing, food processing, drug delivery, gelled rocket propellants, and many others. Whilst atomization of Newtonian fluids, such as water and liquid fuels, is well understood and has been transferred into relatively mature technologies, the physics of non-Newtonian fluids atomization is poorly understood to date, and the literature on this topic is still in its infancy.

Non-Newtonian fluids refer to a broad class of liquids and soft materials with complex microstructure, which can be described by constitutive models where the stress tensor is a non-linear function of the deformation rate tensor. Examples include polymer solutions and melts, particle suspensions, colloidal gels, foams, and emulsion. Although the denomination "non-Newtonian fluids" is widely accepted, it is well known that under certain flow conditions, such as very high shear rates, even Newtonian fluids exhibit a nonlinear response; thus, the "non-Newtonian" attribution is more appropriate for the flow instead of the fluid itself, and the alternative denomination "complex fluids" is preferable.

Constitutive models representative of the most common behaviors observed in non-Newtonian flows describe the macroscopic effect of reversible or irreversible changes in the fluid microstructure, induced by either the applied stress or deformation rate. They can be sorted into time-independent models, where microstructural changes in the fluid occur instantaneously upon shear, and revert to the initial state when shear is removed, and time-dependent models, where the time scale of microstructural changes is not negligible in comparison with the characteristic time of the flow. Time-independent models, such as the Ostwald-de Waele (power law) and the Bingham model, are suitable to describe most of the shear-thinning, shear-thickening and viscoplastic behaviors observed experimentally. Time-dependent models, such as the Maxwell and the Oldroyd-B models, capture several features of viscoelastic fluids.

Atomization of complex fluids is not easy. Whether their microstructure consists of entangled macromolecules or simply of colloidal particles, this results in higher viscosities as compared with aqueous solutions and fuels commonly used in spray applications, which affects the instability and breakup mechanisms of liquid jets and sheets. More importantly, fluids such as most polymeric liquids form stable viscoelastic films and ligaments, which prevent a proper atomization. Thus, in non-Newtonian spray generators the main aerodynamic breakup mechanism is often assisted or enhanced by other methods, such as air injection (effervescent atomizers), swirl injectors, or close-coupled atomization.

This special issue is a collection of four papers on selected aspects of non-Newtonian sprays, with the aim to contribute to filling the current gap in the literature. A comprehensive summary of the existing literature about the formation of drops and sprays from viscoelastic liquids, which shows the limitations of the few models available for the prediction of the drop mean size, is presented by Brenn and Plohl. These authors extend previous studies to provide models for the mean size of drops formed by the breakup of capillary axisymmetric viscoelastic liquid jets and of plane sheets, taking into account the influence of the molecular weight and of the flexibility or rigidity of the polymer molecules on the solution behavior upon deformation through rheometrically accessible stress relaxation and deformation retardation times of the liquid.

A different approach to gain understanding of non-Newtonian sprays relies on numerical simulations. Ertl and Weigand present an investigation of the primary breakup of shear-thinning liquid jets using a Direct Numerical Simulations (DNS) algorithm specifically adapted to simulate free surface flows, and the Carreau-Yasuda constitutive model for the fluid viscosity. This approach enables a systematic, quantitative characterization of shear-thinning on the breakup process, providing an insight into details that are not accessible experimentally.

Whilst these papers focus on the fundamental mechanism of atomization in complex fluids, the work by Negri and Ciezki investigates the influence of elasticity on the atomization of viscoelastic fluids in an impinging jet injector; in particular, they study the breakup morphology of the liquid sheet and of the rim. They identify different spraying regimes, which lead to the construction of diagrams showing the influence of the Weber and Elasticity numbers on 
the morphology; they also propose a physical mechanism to explain how the viscoelastic liquid sheet evolves into ligaments.

One important aspect of spray investigation is the interaction between droplets and the liquid film deposited on the target surface. The work by Blackwell et al. presents an experimental investigation of the impact of viscoplastic (or yield-stress) drops on pre-coated horizontal surfaces, with focus on the effects of drop size, impact velocity, precoating thickness, and rheological material properties. The impact duration, the rebound height, the maximum radial spread, and the final crater diameter are presented as a function of a single dimensionless parameter expressing the ratio of inertial to dissipative forces accounting for the impacting drop geometry.

Although it was not possible to address several important issues concerning non-Newtonian sprays, we hope this special issue will provide a useful reference and, more importantly, inspire further research in this fast-growing and fascinating research subject.

\section{Guest Editor:}

Volfango Bertola

Laboratory of Technical Physics, School of Engineering, University of Liverpool, Liverpool, United Kingdom 